\title{
A pilot study of a metronomic chemotherapy regimen with weekly low-dose docetaxel for previously treated non-small cell lung cancer
}

\section{Takashi Yokoi \\ Takeshi Tamaki \\ Toshiki Shimizu \\ Shosaku Nomura}

First Department of Internal Medicine, Kansai Medical University, Moriguchi City, Osaka, Japan
Correspondence:Toshiki Shimizu First Department of Internal Medicine, Kansai Medical University, I0-15 Fumizono-cho, Moriguchi City, Osaka 570-8506, Japan Tel +8166992 I00I Fax +8I 669948344 Email shimizto@takii.kmu.ac.jp
This article was published in the following Dove Press journal:

Lung Cancer:Targets and Therapy

17 May 2012

Number of times this article has been viewed

Background: Low-dose metronomic (LDM) chemotherapy is a novel approach that involves frequent administration of a low dose of chemotherapeutic agent without a long interval.

Purpose: The aim of this clinical pilot study was to evaluate the toxicity and efficacy of LDM chemotherapy with weekly low-dose docetaxel for previously treated non-small cell lung cancer (NSCLC).

Patients and methods: The enrolled patients received $15 \mathrm{mg} / \mathrm{m}^{2}$ of docetaxel intravenously on a weekly basis without any interval.

Results: Twenty-seven patients were enrolled in the study; 20 were men, and seven were women. The median age was 62 years (range: 32-75). Eleven patients were stage IIIB, and 16 were stage IV. The Eastern Cooperative Oncology Group performance status was 0 or 1. There was no severe hematological adverse effect; importantly, there was no neutropenia or thrombocytopenia. The objective response rate was $7.4 \%$ and the disease control rate was $51.9 \%$. The median survival time was 16.4 months (95\% CI: 5.7-36.4).

Conclusion: Our preliminary results indicate that our metronomic regimen was well tolerated and active in patients with previously treated NSCLC. Thus, further investigation of this LDM regimen is warranted.

Keywords: optimal biological dose, metronomic chemotherapy, docetaxel

\section{Background}

The progress of antitumor chemotherapy over the past few decades has resulted in striking survival benefit for patients with advanced non-small cell lung cancer (NSCLC). However, patients with relapsed NSCLC continue to have poor prognosis. Shepherd et al revealed that salvage chemotherapy with docetaxel led to greater survival benefits than the best supportive care. ${ }^{1}$ Moreover, Fossella et al reported that triweekly docetaxel monotherapy was superior to vinorelbine or ifosfamide for previously treated NSCLC. ${ }^{2}$ These researchers also showed that although overall survival did not differ significantly between docetaxel $100 \mathrm{mg} / \mathrm{m}^{2}$ and docetaxel $75 \mathrm{mg} / \mathrm{m}^{2}$, the docetaxel $75 \mathrm{mg} / \mathrm{m}^{2}$ arm was well tolerated. In Japan, a moderate docetaxel dose $\left(60 \mathrm{mg} / \mathrm{m}^{2}\right)$ was employed because docetaxel $60 \mathrm{mg} / \mathrm{m}^{2}$ demonstrated survival benefits comparable to those obtained with docetaxel $100 \mathrm{mg} / \mathrm{m}^{2} .{ }^{3}$ Based on these findings, triweekly docetaxel therapy has been regarded as standard treatment for previously treated NSCLC.

A novel approach for treating relapsed NSCLC is the use of low-dose metronomic (LDM) chemotherapy. Various preclinical studies have shown LDM chemotherapy might exhibit alternative antitumor effects in addition to the direct cytotoxic effect. ${ }^{4,5,6}$ In LDM chemotherapy, the chemotherapeutic agent can modulate the cancer 
microenvironment and disrupt tumor-associated vascular angiogenesis. This approach involves administering cytotoxic drugs on a daily or weekly basis at low doses without a long interval. Docetaxel has been recognized as an active reagent that can exhibit metronomic activity in vitro and in vivo. ${ }^{6}$ Therefore, we hypothesized that relapsed patients with NSCLC might receive a survival benefit from LDM chemotherapy with docetaxel. In this study, we evaluated the toxicity and efficacy of the novel metronomic regimen in previously treated NSCLC. As far as the authors are aware, this is the first report of a true weekly LDM regimen with docetaxel for relapsed NSCLC.

\section{Patients and methods \\ Patients}

Patients with histologically or cytologically diagnosed NSCLC (clinical stage IIIB or IV at the time of enrollment) who had previously received at least one regimen of systemic chemotherapy were eligible for entry into this study. The clinical stage was assigned based on the sixth edition of the TNM Classification for Lung Cancer. ${ }^{7,8}$ Previous paclitaxel use was allowed. An interval of at least 4 weeks from previous treatment was required. Each patient was required to meet the following criteria: Eastern Cooperative Oncology Group (ECOG) performance status (PS) $0-1$; age $\geq 20$ years old; and adequate organ function as defined by serum creatinine $\leq 1.5 \mathrm{mg} / \mathrm{dL}$, total bilirubin $\leq 1.5 \mathrm{mg} / \mathrm{dL}$, serum aspartate aminotransferase and alanine aminotransferase $\leq 70 \mathrm{IU} / \mathrm{L}, \mathrm{PaO}_{2} \geq 70$ Torr, white blood cells $\geq 3000 / \mu \mathrm{L}$, platelets $\geq 100,000 / \mu \mathrm{L}$, and hemoglobin $\geq 8.0 \mathrm{mg} / \mathrm{dL}$. Patients with another clinically active malignancy were excluded. The study was carried out according to the Declaration of Helsinki and was approved by the Clinical Research Board of Kansai Medical University, Takii Hospital. Written informed consent was obtained from all patients.

\section{Treatment}

Docetaxel $\left(15 \mathrm{mg} / \mathrm{m}^{2}\right)$ diluted with $5 \%$ glucose $(250 \mathrm{~mL})$ was administered intravenously over 60 minutes each week. The treatment was carried out on a true weekly basis without any interval. To prevent a hypersensitivity reaction, $2 \mathrm{mg}$ of dexamethasone or H1-blocker was given as premedication to all patients. The treatment was repeated unless disease progression was observed. Response was evaluated with chest computer tomography every month during the treatment period. Administration of docetaxel could be skipped if the patient experienced unacceptable toxicity (eg, grade 3 or worse hematological/nonhematological toxicity, not appetite loss, constipation, and nausea/vomiting). Patients who did not wish to continue the regimen and those showing a decline in PS (PS 4) were allowed to withdraw from the study.

\section{Statistical analysis}

The primary endpoint of this study was feasibility. The secondary endpoints were overall survival time (OS) and objective response rate (ORR). The OS was defined as the time from the start of the LDM chemotherapy to the time of death from any cause or to the date the patient was last known to be alive. Treatment-related adverse effects were evaluated using National Cancer Institute Common Toxicity Criteria, version 2.0. ${ }^{9}$ Objective tumor responses were evaluated using the Response Evaluation Criteria in Solid Tumors, version 1.0. ${ }^{10}$ The ORR was defined as the proportion of complete remission (CR) and partial remission (PR) compared with the total number of patients. The disease control rate (DCR) was defined as the proportion of CR, PR, and stable disease (SD) compared with the total number of patients. The minimal time interval required between two measurements for determining SD was 6 weeks. The $95 \%$ confidence interval $(95 \% \mathrm{CI})$ for the ORR and the DCR was calculated using the binomial distribution. The OS was estimated with the Kaplan-Meier product-limit method. ${ }^{11}$ The $95 \%$ CIs of the survival rate were calculated using Greenwood's method. ${ }^{12}$ To calculate the $95 \%$ $\mathrm{CI}$ of the median survival time (MST), the Brookmeyer and Crowley method was used. ${ }^{13}$ All statistical analyses were conducted using the JMP9 version 9.0.2 Program for Windows (SAS Institute Inc, Cary, NC).

\section{Results \\ Patient characteristics}

From June 2000 to September 2005, 27 patients were enrolled in this study. The patients' characteristics are summarized in Table 1. All the patients were Japanese and the median age was 62 years (range: 32-75). There were seven women and 20 men in the study. Adenocarcinoma and squamous cell carcinoma were present in 17 and ten patients, respectively. Eleven patients had stage IIIB disease, while 19 patients had stage IV disease. The Eastern Cooperative Oncology Group PS was 0 and 1 in 13 and 14 patients, respectively. All patients had received cytotoxic chemotherapy, eleven patients had received thoracic radiotherapy, and five patients had undergone surgery. Previous chemotherapy consisted of one regimen in 14 patients, two regimens in twelve patients, and three regimens in one patient. The median number of cycles administered to 27 patients was 20 (range: 2-60). The reasons for withdrawal from this regimen were disease 
Table I Patient demographics and disease characteristics at baseline

\begin{tabular}{ll}
\hline Number of patients & $\mathbf{n}(\%)$ \\
\hline Gender & $\mathbf{2 7}$ \\
Male & 20 \\
Female & 7 \\
Age (years) & \\
Median & 62 \\
Range & $32-75$ \\
ECOG performance status & \\
0 & $13(48.1)$ \\
I & $14(51.9)$ \\
Histology (cytology) & $17(63.0)$ \\
Adenocarcinoma & $10(37.0)$ \\
Squamous cell carcinoma & \\
Disease stage at study entry & $11(40.7)$ \\
IIIb & $16(59.3)$ \\
IV & \\
Previous treatments & 27 \\
Systemic chemotherapy & 11 \\
Thoracic radiotherapy & 5 \\
Surgery & \\
Number of prior systemic therapies & 14 \\
I regimen & 12 \\
2 regimen & 1 \\
$\geq 3$ regimens & 617 \\
Total number of cycles administered & 20 \\
Median & $2-60$ \\
Range &
\end{tabular}

Abbreviation: ECOG, Eastern Cooperative Oncology Group.

progression (16), unacceptable toxicity (five), deterioration of PS (three), and refusal to continue the treatment (three). After withdrawing from this study, nine patients received at least one chemotherapy regimen including epidermal growth factor receptor tyrosine kinase inhibitor.

\section{Treatment-related adverse effects}

The treatment-related adverse effects observed in this study are summarized in Table 2. No treatment-related death was observed. Grade 3 or 4 neutropenia and thrombocytopenia were not observed. Grade 3 or 4 anemia was observed in two patients (7.4\%). Pleural effusion was the most common nonhematological adverse effect observed in this study. Pleural effusion of grades $1-3$ was observed in 16 patients $(59.3 \%$, 95\% CI: 38.8-77.6), of whom two patients (7.4\%) showed grade 3 pleural effusion. Edema in extremities was observed in five patients $(18.5 \%, 95 \%$ CI: 6.3-38.1). Grade 1 diarrhea was observed in two patients, but grade 2 or worse diarrhea was not observed. Other observed hematological or nonhematological adverse effects were mild and manageable. Thus, these findings suggest that the LDM docetaxel regimen employed in this study was feasible.
Table 2 Treatment-related toxicity profiles

\begin{tabular}{lllll}
\hline & $\begin{array}{l}\text { Grade I } \\
\text { n (\%) }\end{array}$ & $\begin{array}{l}\text { Grade 2 } \\
\text { n (\%) }\end{array}$ & $\begin{array}{l}\text { Grade 3 } \\
\text { n (\%) }\end{array}$ & $\begin{array}{l}\text { Grade 4 } \\
\text { n (\%) }\end{array}$ \\
\hline Anemia & I4 (5I.9) & $7(25.9)$ & $\mathrm{I}(3.7)$ & $\mathrm{I}(3.7)$ \\
Neutropenia & $\mathrm{I}(3.7)$ & $4(14.8)$ & - & - \\
Thrombocytopenia & $3(11.1)$ & - & - & - \\
Hepatotoxicity & $\mathrm{I} 4(51.9)$ & - & - & - \\
Nephrotoxicity & $3(11.1)$ & - & - & - \\
Diarrhea & $2(7.4)$ & - & - & - \\
Pleural effusion & $12(44.4)$ & $2(7.4)$ & $2(7.4)$ & - \\
Edema & $2(7.4)$ & $3(11.1)$ & - & - \\
\hline
\end{tabular}

\section{Clinical outcomes}

The objective responses are summarized in Table 3. One patient showed CR; one, PR; ten, SD; and 15, progressive disease. The ORR and DCR were 7.4\% (95\% CI: 0.9-24.3) and $51.9 \%$ (95\% CI: 32.0-71.3), respectively. Twenty-two of the 27 patients died, and five patients were censored due to lack of follow-up. The Kaplan-Meier survival curve of OS is shown in Figure 1. The MST was 16.4 months (95\% CI: 5.7-36.4). The 1-, 2-, and 5-year survival rates were 58.8\% (95\% CI: 40.1-77.5), 47.1\% (95\% CI: 27.9-66.2), and 12.1\% (95\% CI: 0-26.9), respectively. The median progression-free survival time (PFS) was 6.5 months (95\% CI: 3.4-11.0). However, the PFS data are for reference purposes only because PFS was not included among the study endpoints.

\section{Discussion}

Preclinical data clearly demonstrated that LDM chemotherapy could exhibit an alternative antitumor effect in addition to the direct cytotoxic effect. Browder et al showed that LDM chemotherapy with cyclophosphamide eradicated Lewis lung carcinoma, which was refractory to conventional maximum tolerated dose (MTD) chemotherapy. ${ }^{14}$ Kamat et al also described that compared with MTD docetaxel, LDM docetaxel treatment against a human ovarian cancer xenograft model exhibited tumor reduction, leading to improved survival. ${ }^{6}$ Metronomic docetaxel has also been shown to decrease the microvessel density of tumors and inhibit the mobilization of circulating endothelial precursors. Thus, these findings have been the basis for several clinical studies on the use of LDM chemotherapy for NSCLC treatment. ${ }^{15,16}$

Table 3 Summary of objective tumor responses

\begin{tabular}{ll}
\hline & $\mathbf{n}(\%)$ \\
\hline Complete remission & $\mathrm{I}(3.7)$ \\
Partial remission & $\mathrm{I}(3.7)$ \\
Stable disease & $12(44.4)$ \\
Progressive disease & $13(48.1)$ \\
\hline
\end{tabular}




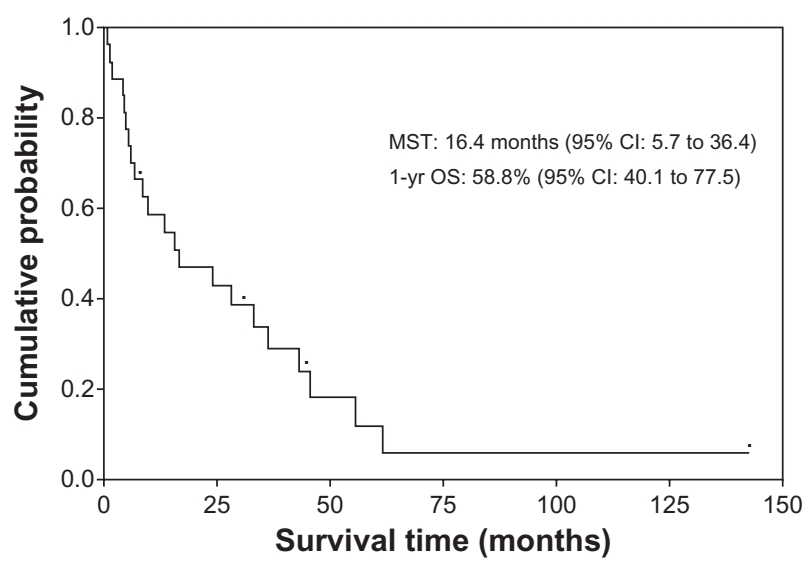

Figure I Kaplan-Meier curve of OS for patients enrolled in this study. Note: The median survival time was 16.4 months (95\% Cl: 5.7-36.4).

Abbreviations: $\mathrm{Cl}$, confidence interval; MST, median survival time; OS, overall survival.

Based on the metronomic theory, drug administration should be repeated as frequently as possible and without any interval. Therefore, we employed a true weekly scheduling scheme. Hainsworth et al reported a Phase I study involving a true weekly docetaxel regimen for patients with refractory solid tumor. ${ }^{17}$ They proposed an MTD of $20 \mathrm{mg} / \mathrm{m}^{2} /$ week when used concurrently with radiation therapy. Patients with relapsed NSCLC often require palliative radiation therapy against brain or bone metastasis. Therefore, for this study, we determined the study dose of docetaxel based on Hainsworth et al's Phase I study.

Dose setting in LDM chemotherapy should be determined based on the optimal biological dose (OBD) instead of the MTD. ${ }^{5}$ However, it is difficult to determine the OBD because there is no accurate surrogate marker for LDM chemotherapy. Tas et al evaluated the effect of the MTD and LDM chemotherapy on the concentrations of serum vascular endothelial growth factor, thrombospondin-1, and vascular endothelial growth factor receptor 1 in patients with advanced NSCLC. ${ }^{18}$ The results revealed that angiogenic hallmarks do not always serve as surrogate markers for determining the OBD in LDM chemotherapy. However, LDM chemotherapy could also promote the differentiation of tumor cells. Eckert et al and Grünberg et al observed that docetaxel promoted the differentiation of human colon carcinoma cell line HT-29 in vitro at low concentrations rather than high concentrations, leading to the inhibition of cell proliferation. ${ }^{19,20}$ Apart from the antiangiogenic effect, the previously mentioned direct modulation of tumor phenotypes by LDM chemotherapy requires a distinct surrogate marker, other than angiogenic hallmarks, to determine the OBD. To date, there is no accurate surrogate marker for deciding the OBD in LDM chemotherapy.

In general, the results show that our novel LDM docetaxel chemotherapy approach is feasible. In particular, the hematological adverse effects were extremely mild and the prevalence and grade of neutropenia were strikingly lower than those reported previously. ${ }^{1,2,3,21}$ Our LDM docetaxel regimen did not result in grade 3 or 4 neutropenia, whereas conventional triweekly $75 \mathrm{mg} / \mathrm{m}^{2}$ docetaxel led to the development of grade 3 or 4 neutropenia in $40.2 \%$ of patients. ${ }^{21}$ In addition, the prevalence of diarrhea was significantly decreased. The prevalence of diarrhea in the LDM docetaxel and MTD docetaxel regimens was $7.4 \%$ and $24.3 \%$, respectively. The well-tolerated toxic profiles of our LDM regimen should enable patients to receive long-term treatment without an interval.

Fluid retention, characterized by peripheral edema and nonmalignant pleural effusion, is a specific adverse effect of docetaxel. An increase in vascular permeability and subsequent capillary protein leak have been regarded as pathological etiologies of fluid retention. ${ }^{22}$ However, a previous report showed pleural effusion occurred preferentially in weekly docetaxel rather than in triweekly docetaxel regimens. ${ }^{23}$ In our study, the most common nonhematological adverse effect was pleural effusion with this LDM regimen. Unfortunately, we did not always conduct cytological examinations of the pleural effusions, so we could not determine whether the pleural effusion was definitely due to the LDM docetaxel regimen. In addition, we employed premedication with $2 \mathrm{mg}$ of dexamethasone. This premedication with a relatively low dose of corticosteroid may have led to frequent pleural effusion. We tried to manage patients who developed pleural effusion using oral diuretics. However, the PS of three patients decreased due to refractory pleural effusion, resulting in withdrawal from the study. Thus, careful management of fluid retention is necessary when employing this LDM regimen.

The results from three large-scale randomized studies demonstrated that the ORR and DCR of a $75 \mathrm{mg} /$ $\mathrm{m}^{2}$ triweekly docetaxel regimen for previously treated NSCLC were approximately $5.5 \%-8.8 \%$ and $52 \%-55.2 \%$, respectively. The results from our LDM regimen (ORR: 7.4\%; DCR: $51.9 \%$ ) were highly comparable to those of previous studies. ${ }^{1,2,21}$ In addition to the comparable tumor responses, our LDM regimen yielded a highly favorable OS and showed an MST of 16.4 months, whereas previous studies reported values of 5.7-7.9 months. Lielenbaum et al 
reported a Phase II study of weekly docetaxel containing $36 \mathrm{mg} / \mathrm{m}^{2} /$ week for 6 consecutive weeks every 8 weeks. ${ }^{24}$ The study included PS 2 patients. The MST for the subgroup of PS 0 or 1 was 11.9 months. Our LDM regimen data were also better than theirs. In addition, the hematological toxicity profile of our regimen was better than theirs. However, fluid retention was more frequent and more severe in our regimen.

A recent report noted that adenocarcinoma has better prognostic predisposition than squamous cell carcinoma. ${ }^{25}$ We examined an additional analysis for histological subtype. The MST in the squamous cell carcinoma group and the adenocarcinoma group was 5.5 months and 33.0 months, respectively. There was a significant difference in OS $(P=0.0031)$. Moreover, the MST of the adenocarcinoma group was much better than previous reports. Natural long survivors may have selectively accumulated in the adenocarcinoma group leading a better survival profile. Altogether, we cannot deny the possibility that our LDM regimen may benefit a particular subpopulation, especially patients with adenocarcinoma, rather than all relapsed NSCLC patients. Despite the small size of this study, these findings suggest our LDM regimen could partially replace the conventional triweekly regimen.

\section{Conclusion}

In this study, we reported the results of a pilot study of a novel metronomic treatment schedule involving weekly low-dose docetaxel therapy in patients with previously treated NSCLC. The treatment was well tolerated and active. Therefore, this metronomic regimen is a promising alternative treatment option in previously treated NSCLC patients and warrants further investigation in Phase III studies.

\section{Disclosure}

The authors declare no conflicts of interest in this work.

\section{References}

1. Shepherd FA, Dancey J, Ramlau R, et al. Prospective randomized trial of docetaxel versus best supportive care in patients with non-small-cell lung cancer previously treated with platinum-based chemotherapy. J Clin Oncol. 2000;18(10):2095-2103.

2. Fossella FV, DeVore R, Kerr RN, et al. Randomized phase III trial of docetaxel versus vinorelbine or ifosfamide in patients with advanced non-small-cell lung cancer previously treated with platinum-containing chemotherapy regimens. The TAX 320 Non-Small Cell Lung Cancer Study Group. J Clin Oncol. 2000;18(12):2354-2362.

3. Mukohara T, Takeda K, Miyazaki M, Takifuji N, Terakawa K, Negoro S. Japanese experience with second-line chemotherapy with low-dose $\left(60 \mathrm{mg} / \mathrm{M}^{2}\right)$ docetaxel in patients with advanced non-small-cell lung cancer. Cancer Chemother Pharmacol. 2001;48(5):356-360.
4. Hahnfeldt P, Folkman J, Hlatky L. Minimizing long-term tumor burden: the logic for metronomic chemotherapeutic dosing and its antiangiogenic basis. J Theor Biol. 2003;220(4):545-554.

5. Shaked Y, Emmenegger U, Man S, et al. Optimal biologic dose of metronomic chemotherapy regimens is associated with maximum antiangiogenic activity. Blood. 2005;106(9):3058-3061.

6. Kamat AA, Kim TJ, Landen CN Jr, et al. Metronomic chemotherapy enhances the efficacy of antivascular therapy in ovarian cancer. Cancer Res. 2007;67(1):281-288.

7. Mountain CF. Revisions in the International System for Staging Lung Cancer. Chest. 1997;111(6):1710-1717.

8. Sobin L, Wittekind CH, eds. TNM Classification of Malignant Tumours, Sixth Edition. New York: Wiley-Liss, 2002:99-103.

9. Oken MM, Creech RH, Tormey DC, et al. Toxicity and response criteria of the Eastern Cooperative Oncology Group. Am J Clin Oncol. 1982; 5(6):649-655.

10. Therasse P, Arbuck SG, Eisenhauer EA, et al. New guidelines to evaluate the response to treatment in solid tumors. European Organization for Research and Treatment of Cancer, National Cancer Institute of the United States, National Cancer Institute of Canada. J Natl Cancer Inst. 2000;92(3):205-216.

11. Kaplan EL, Meier P. Nonparametric estimation from incomplete observations. J Am Stat Assoc. 1958;282(53):457-481.

12. Greenwood M. The natural duration of cancer. Reports on Public Health and Medical Subjects. Her Majesty's Stationery Office, London. 1926;33: $1-26$.

13. Brookmeyer R, Crowley J. A confidence interval for the median survival time. Biometrics. 1982;38:29-41.

14. Browder T, Butterfield CE, Kräling BM, et al. Antiangiogenic scheduling of chemotherapy improves efficacy against experimental drug-resistant cancer. Cancer Res. 2000;60(7):1878-1886.

15. Görn M, Habermann CR, Anige M, et al. A pilot study of docetaxel and trofosfamide as second-line "metronomic" chemotherapy in the treatment of metastatic non-small cell lung cancer (NSCLC). Onkologie. 2008;31(4):185-189.

16. Sanborn SL, Cooney MM, Dowlati A et al. Phase I trial of docetaxel and thalidomide: a regimen based on metronomic therapeutic principles. Invest New Drugs. 2008; 26(4):355-362.

17. Hainsworth JD, Burris HA 3rd, Erland JB, Thomas M, Greco FA. Phase I trial of docetaxel administered by weekly infusion in patients with advanced refractory cancer. J Clin Oncol. 1998;16(6):2164-2168.

18. Tas F, Duranyildiz D, Soydinc HO, et al. Effect of maximum-tolerated doses and low-dose metronomic chemotherapy on serum vascular endothelial growth factor and thrombospondin-1 levels in patients with advanced nonsmall cell lung cancer. Cancer Chemother Pharmacol. 2008;61(5):721-725.

19. Eckert K, Fuhrmann-Selter T, Maurer HR. Docetaxel enhances the expression of E-cadherin and carcinoembryonic antigen (CEA) on human colon cancer cell lines in vitro. Anticancer Res. 1997;17(1A): 7-12.

20. Grünberg E, Eckert K, Maurer HR. Docetaxel treatment of HT-29 colon carcinoma cells reinforces the adhesion and immunocytotoxicity of peripheral blood lymphocytes in vitro. Int J Oncol. 1998;12(4): 957-963.

21. Hanna N, Shepherd FA, Fossella FV, et al. Randomized phase III trial of pemetrexed versus docetaxel in patients with non-small-cell lung cancer previously treated with chemotherapy. J Clin Oncol. 2004;22(9): 1589-1597.

22. Semb KA, Aamdal S, Oian P. Capillary protein leak syndrome appears to explain fluid retention in cancer patients who receive docetaxel treatment. J Clin Oncol. 1998;16(10):3426-3432.

23. Piccart MJ, Klijn J, Paridaens R, et al. Corticosteroids significantly delay the onset of docetaxel-induced fluid retention: final results of a randomized study of the European Organization for Research and Treatment of Cancer Investigational Drug Branch for Breast Cancer. $J$ Clin Oncol. 1997;15(9):3149-3155. 
24. Lilenbaum RC, Schwartz MA, Seigel L, et al. Phase II trial of weekly docetaxel in second-line therapy for nonsmall cell lung carcinoma. Cancer. 2001;92(8):2158-2163.
25. Scagliotti G, Brodowicz T, Shepherd FA, et al. Treatment-by-histology interaction analyses in three phase III trials show superiority of pemetrexed in nonsquamous non-small cell lung cancer. J Thorac Oncol. 2011;6(1):64-70

\section{Publish your work in this journal}

Lung Cancer: Targets and Therapy is an international, peer-reviewed, open access journal focusing on lung cancer research, identification of therapeutic targets and the optimal use of preventative and integrated treatment interventions to achieve improved outcomes, enhanced survival and quality of life for the cancer patient. Specific topics covered in the journal include:
Epidemiology, detection and screening; Cellular research and biomarkers; Identification of biotargets and agents with novel mechanisms of action; Optimal clinical use of existing anticancer agents, including combination therapies; Radiation and surgery; Palliative care; Patient adherence, quality of life, satisfaction; Health economic evaluations.

Submit your manuscript here: http://www.dovepress.com/lung-cancer-targets--therapy-journal 